\title{
Searching Evidences of Stroke in Animal Models: A Review of Discrepancies
}

\author{
Ahmet Hilmi KAYA ${ }^{1}$, Hakan ERDOGAN², Erol TASDEMIROGLU ${ }^{1}$ \\ ${ }^{1}$ Anadolu Medical Center, Department of Neurological Sciences, Istanbul, Turkey \\ ${ }^{2}$ Maltepe University, Faculty of Medicine, Department of Neurosurgery, Istanbul, Turkey
}

\section{ABSTRACT}

So far, animal models have helped us better understand the pathophysiology of the ischemic brain damage but they could not contribute so much to clinical practice. The discrepancies in results regarding neuroprotective agents in animal experiments compared to clinical trials have not been solved. Various animal models of ischemic stroke have proven efficacy of many neuroprotective agents without any considerable result in phase III clinical trials.

As is well known, stroke-related focal cerebral ischemia or cardiac arrest related global cerebral ischemia are major causes of disability and death among human subjects. Animal models are essential to evaluate the therapeutic approaches for humans.

In this review, we will try to answer two important questions: 1) Which factors endanger the reliability of experimental studies of stroke on animal models? 2) How can we design our experiments to reflect the neurorestoration and/or neuroprotection mechanisms following ischemic injury, when it comes to human disease?

KEYWORDS: Stroke, Animal models, Discrepancies

\section{LITERATURE REVIEW METHODS}

$\mathrm{O}$ ur review method was based on articles dealing with the relations between animal models and clinical trials. By performing a PubMed search, we aimed to identify all relevant papers to call attention to the discrepancy between experimental methodology in animal modeling and clinical experiences. Articles that were referenced by reviewed articles were also evaluated. Data from papers were extracted within the following categories: study design, type of study population, sampling strategy. Articles excluded from the review were studies that were describing surgical techniques, experimental design and methodology.

\section{AN OVERVIEW OF STROKE MODELS}

The human adult brain consumes about $8 \mathrm{cal} / 100 \mathrm{gr} / \mathrm{min}$ energy. Normally, almost $100 \%$ of glucose is metabolised, but if starved this increases up to $30 \%$, and glucose consumption can be replaced with ketone bodies. In total starvation such as global cerebral ischemia, the brain's limited reserves of glycogen and glucose are barely able to fuel normal brain activity up to 5 minutes. It is reported that the energy stores of the brain are equivalent to $20 \mathrm{cl} / 100 \mathrm{gr}$ (39). In a healthy person, cerebral blood flow (CBF) is autoregulated at $45-50$ $\mathrm{ml} / 100 \mathrm{gr} / \mathrm{min}$ with a mean arterial blood pressure (MABP) of $60-130 \mathrm{~mm} / \mathrm{Hg}$ (Figure 1). In the cerebral ischemia model, the investigators try to reduce the oxygen and glucose supply of cerebral tissue. This process produces ischemic brain injury via a variety of cellular and molecular mechanisms.

There are three major types of ischemic stroke models: global, focal and lacunar. The global ischemic model is produced by occlusion of four (both vertebral and carotid arteries) or two vessels (either both carotid or vertebral arteries) with or without concomittant hypotension during ischemia period (38). In this model, global blood flow is ceased completely, which resembles the condition of cardiac arrest or coronary 
artery occlusion in humans and is mainly used to produce hippocampal damage. This is a minimally invasive ischemia model that enables investigators to study neuronal cell death, apoptosis and reactive gliosis etc. Therefore traumatic effects in the focal ischemia model due to craniotomy are avoided in the global cerebral ischemia model. In the global ischemia model, animals can survive months or even years with minimal veterinary care; whereas in focal cerebral ischemia models, animals encounter significant morbidity and require constant veterinary care throughout the survival period. Mainly, the minimally invasive global ischemia model accomplishes most of the pathological manifestations of neuronal cell death including $90 \%$ cell loss in the hippocampus and $50-65 \%$ cell loss in the caudate, putamen and cortex (41). However, in focal cerebral ischemia models, mainly the caudate, putamen and cortex show neuronal cell loss.

The prenatal/neonatal hypoxia-ischemia stroke models are also considered in global cerebral ischemia (32). Each of these models allows a different degree of reperfusion after a period of ischemia. The most widely used model of neonatal asphyxial brain injury, the 7-day-old rat, in many ways has brain maturity equivalent to that of an early third trimester human fetus (4). Fetal sheep exposed to maternal hypoxemia (17) or to umbilical cord occlusion (16) are also the most widely utilized models of intrauterine hypoxemia-ischemia. Recently, a new model of perinatal brain injury global hypoxemia-ischemia is the near-term fetal rabbit, which avoids most of the problems associated with fetal lamb model. This new model very nicely mimics acute placental insufficiency in humans, and surviving newborn rabbits that display persistent hypertonia and motor deficits provide a striking phenotype of cerebral palsy (6).

Since neuronal plasticity is effective in the newborn brain, many studies of neonatal ischemia-hypoxia are designed for long-term follow-up and functional outcome that include tests of learning memory, coordination, cognition, behavior and motor function at later time points. These studies now enable investigators to determine 1) whether the model resembles the injury of newborn humans; 2) whether potential therapies alter neuropathology, biochemical, and functional outcomes; and 3) whether late neuropathology and functional outcomes correlate with one another.

Most focal cerebral ischemia models involve occlusion of one major blood vessel such as the middle cerebral artery (MCA) (15). In this model, there may be absolutely no blood flow in the very central core of the ischemia, but there is some flow that reaches the area with collateral circulation. MCA occlusion (MCAO) results in a reduction of CBF in both the striatum and cortex (15). Several different types of MCAO models exist, and for the most part they are either permanent or temporary (reperfusion) occlusion with MCA. These MCAO models have been used extensively since they resemble human thromboembolic stroke (20). Experimental models are also available to study ischemic posterior circulation stroke (28). Although the mechanism and the clinical management are different, the posterior circulation stroke model is also included in the focal cerebral ischemia models (28).
Lacunar strokes are small subcortical strokes caused by occlusion of single penetrating arteries. The key mechanisms of lacunar infarcts are microatheroma and lipohyalinosis (5). Around $25 \%$ of all ischemic strokes are lacunar infarcts (33). Although they have an apparently good functional prognosis compared with focal ischemic stroke, lacunar strokes cause substantial physical disability and dementia. Inadequately, little attention has been given to specific treatments and animal models of lacunar infarcts (33). In animal models, lacunar stroke is created by either microthrombi or microsphere injection into the internal carotid artery (1). Besides, endothelin injection into the deep gray matter is performed to create a lacunar infarct (1). Rabbits, mice or rats are used for this model (33). Despite this effort, it is unclear which animal model of lacunar stroke is the most appropriate. Treatments preventing further lacunar strokes would be useful although a few studies tested treatments administered before stroke induction (33). However, lacunar stroke models are not so reliable when compared to global ischemia models.

There are three main targets for stroke treatment: 1) Stroke prevention-prophylaxis (during pre- or post-stroke period), 2) Neuroprotection and 3) Neurorestoration (18). So far, the current therapy of stroke is limited with the exception of thrombolysis to tissue plasminogen activator (TPA) therapy (2). Thus, two different types, acute and chronic experiments are utilized for experimental cerebral ischemia. If the therapeutic agent is considered for neuroprotection, the therapeutic window will be likely within the acute stage of stroke. If the therapeutic agent has neurorestorative effects, the therapeutic window will be the subacute and chronic stage of stroke. While the neuroprotection studies require a therapeutic window within the few to several hours, the therapeutic window for initial treatment in neurorestoration studies extends to days, weeks or even months after the ischemic episode. Failure of translating laboratory products to the clinic may occur due to the discontinuity of treatments that transgress from the acute, to the subacute and chronic phases of stroke in preclinical studies. Therapies of neuroprotection, neurorestoration and stem cell transplantation should be evaluated seriously in this regard (38).

\section{- PARAMETERS AFFECTING OUTCOME}

In the cerebral ischemia model, the main purpose is to create a minimally invasive stroke model that has the most reliable infarct size for each animal. Each model requires care in evaluating main physiological conditions such as blood pressure, brain and body temperature, blood gases, blood counts, CBF and finally consistent and reproducible infarction size or neuronal injury. Number of animals, gender, age and weight should also be the same. However, some other factors should be taken into consideration while working on stroke.

Mainly, strokes produced in the animal models differ from the strokes which occur in humans. There are multiple co-morbid conditions in human stroke. These are age, hypertension, diabetes, obesity, hyperlipidemia, cardiovascular diseases, sex difference, patients' medications etc. Since these factors that result in pathology are not considered in the animal 
stroke models, novel therapies will not closely approximate the clinical setting.

Age: As a great risk factor for stroke, the process of aging results in many inflammatory changes. Continuous antigenic load and stress leads to the phenomenon, "inflamm-aging", which is also related with ischemic stroke (13). The inflammation increases tendency to stroke and predisposes development of the glial scar and damaging response after stroke as well. The Blood-Brain Barrier (BBB) protects the neural tissue from harmful substances. Aging is associated with disruption of BBB and the influx of inflammatory cells following stroke (7). In the elderly population, efficacy and safety profiles of many stroke medications interact with the spontaneous recovery and risk of hemorrhagic transformation due to thrombolytic therapy. Most of the small animal (rodent) stroke models utilize young animals. These young animals have more prominent brain plasticity and they show better spontaneous recovery by improving response to neuroprotective and neurorestorative treatments after an experimental insult. There are very few studies that involve older animals with stroke (29). However, accurate evaluation of functional parameters and potential therapeutics truly relevant to the clinic necessitates standardization of the studies according to age (38).

Hypertension: This is the most prevalent risk factor for stroke that not only causes aberrant cerebrovascular response to ischemia but also leads to compensatory alterations of the neurovascular unit. Therapeutic agents generated from nonhypertensive animal experiments should target the nonhypertensive patients. However, these therapeutic agents should be considered in hypertensive rats.

Diabetes: Diabetes and hyperglycemia occur in one-third of acute stroke patients and cause slowdown of recovery. The close interaction between diabetes and the prognosis

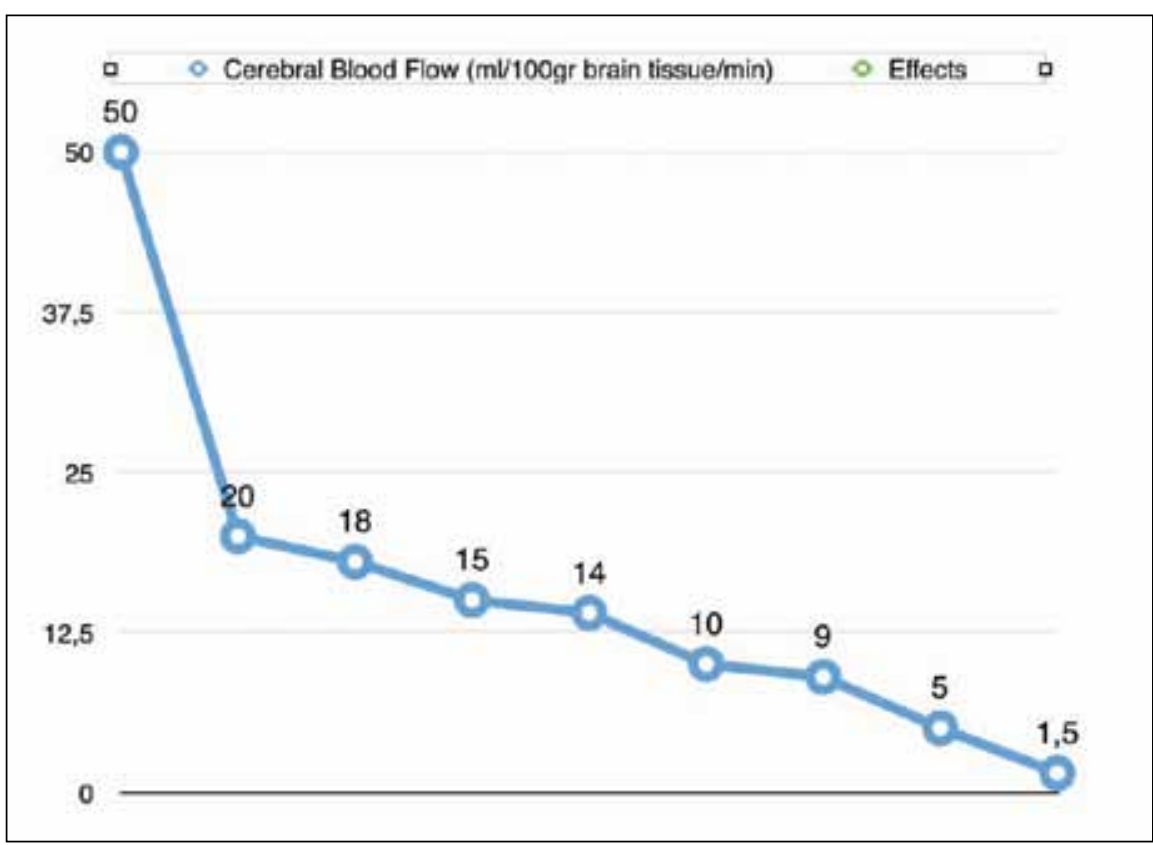

\begin{tabular}{|r|l|}
\hline $\begin{array}{l}\text { Cerebral Blood } \\
\text { Flow }(\mathrm{ml} / 100 \mathrm{gr} \\
\text { brain tissue/min })\end{array}$ & Effects \\
\hline 50 & Normal \\
20 & Tolerable, limit of extraction of oxygen and ischemia \\
18 & Spontaneous seizure activity \\
15 & EEG flattens \\
14 & Somatosensorial evoked potentials could not be recorded \\
\hline 10 & Sudden membran depolarization of neurons \\
9 & Irreversible effects of cells \\
5 & Nerve cells lose their metabolic capacity to transport ion \\
1,5 & Infarction \\
\hline & \\
\hline
\end{tabular}

Figure 1: Changes in cerebral blood flow according to the phases of stroke. 
of recanalization after ischemia requires using diabetic and/ or hyperglycemic animals as a stroke model, especially when thrombolytic therapeutics are tested. Reduced thrombolytic efficacy and an increased post-ischemic hemorrhage incidence are closely associated with post-stroke hyperglycemia and diabetes mellitus (10).

Obesity: Obesity was supposed to be casually related to stroke, indicating the need to some other risk factors such as hypertension, hyperlipidemia, and glucose intolerance. But a large prospective study has demonstrated association between body mass index and both ischemic and haemorrhagic stroke. Song et al. have suggested that individuals with a body mass index above the reference category of 22-23 were at increased risk (35).

Hyperlipidemia: There is accumulating evidence about the role of hyperlipidemia as a comorbid factor in stroke. Besides, hyperlipidemia exacerbates the host immune responses after ischemia by aggravating the cerebral ischemia. Many studies the examine novel therapies in stroke animals with hyperlipidemia are being conducted but the lack of laboratory investigations on these animals still remains (38). High-quality studies are needed for evaluating neurorestoration in stroke.

Heart Disease: Without a doubt, heart disease increases the risk of stroke directly or indirectly. We may suggest that therapeutics currently used in stroke may also play role in recovery from cardiac diseases. There have been limited studies investigating the common co-morbid conditions of these two entities (38).

Sex Difference: It is well known that pre-menopausal women have a lower incidence of stroke compared with men throughout most of the lifespan which has been ascribed to protective effects of gonadal steroids, most notably estrogen (26). The ovarian hormone $17 \beta$-estradiol (E2) exerts profound neuroprotective actions against brain damage induced by global and focal ishemia in rodent models of ischemia (22). However, as women age, they disproportionately get affected by stroke. Coincident with the loss of estrogen after menopause, the stroke risk in elderly women increases. Decreased ischemic damage occurs in adult female vs. male rodents in many models of induced global and focal cerebral ischemia (19). Hormone levels are similar in males and females prior to puberty yet females show reduced ischemic damage. These findings suggest that there must be an unknown interaction with age and hormone loss or an intrinsic sex difference mediated by chromosome compliment and/or organizational effects of steroids (19).

There are experimentally controllable physiological parameters that affect the outcome. Several studies have shown that training of the animals before experiments reduces the infarct volume and improves functional recovery in animal models of stroke (34). Forced physical training and skilled reaching training were identified as the most effective training strategies. It is assumed that training augments endogenous repair mechanisms following stroke (27). It is also stated that the stressful component of forced paradigms may elevate the serum levels of glucocorticoid hormones and thereby exac- erbate ischemic injury. However, voluntary physical training, which mostly involves optional wheel running, produces less stress (25).

\section{- DISCREPANCIES}

In 2004, Macleod et al. reported that more than 4000 papers investigating at least 700 neuroprotective drugs have been published (30). Still, none of these drugs seems to be accepted for treatment of acute stroke patients.

Most stroke victims take medication for hypertension, diabetes mellitus, hyperlipidemia and cardiovascular disease. However, new therapeutics have been evaluated together with drugs against these factors in very few studies (38). The complexities originating from interactions between these pathologies have to be understood for development of new therapies. Until now, we have failed to evaluate the risk factors such as hypertension and diabetes that play important roles in ischemic sroke (11). According to report of Howells et al. (21), among the 3142 animal experiments on neuroprotection published in medical literature up to 2010 , only $11 \%$ involved testing in hypertensive and only $1 \%$ in diabetic animals.

Most studies use only male animals to avoid the interference of the hormone cycle. However, both male and female patients are treated for stroke in clinical practice. Males and females differ from each other in their cellular pathways during stroke damage and this could be another reason for distinction between the treatment strategies (40).

When we compare animal and clinical studies for acute stroke therapies, we encounter some potential problems and pitfalls as follows:

A) Animal studies are carried out with young animals without co-morbid conditions. Besides, these studies are performed under anesthesia, require a surgical procedure for vessel occlusion and lead to a modification of confounding factors. Investigators have the opportunity to arrange the time between onset of ischemia and drug administration. However, physicians have to deal with elderly stroke patients with severe concomitant diseases in busy emergency rooms. The conditions provided for an experimental study are far from simulating the ones of a physician who tries to balance the problems such as diabetes, hypertension or heart failure as well as to evaluate and treat the ischemic cerebral damage as soon as possible (23).

B) In clinical stroke trials, too many mild or severe patients are included in the study so protocol violations take place. Many clinical trials are initiated with insufficient preclinical data of the patients. Furthermore, most of the animal studies are not performed in strictly randomised, double-blind fashion.

C) In animal studies, prolonged survival and neurological improvement rates are not documented realistically. Histopathological findings and treatment effects are rarely adequate to reveal the mechanisms in behavioral and functional improvement. There is great difference between animal experiments and clinical practice in terms of outcome evaluation. The cerebral infarct area is used in animal experiments while 
neurological function and quality of life are more important in humans.

D) In experimental studies, the drug treatment appropriate time window is used for drug administration. It is started before or very early after induction of ischemia, which is not relevant to clinical conditions. In clinical studies, the appropriate time window is not used and adequate drug levels may not be achieved because of toxicity.

Mostly small species, like rodents, have been used for testing all stages of stroke (38). Small animal care following experimental stroke is easier than large animals' postoperative care.

Rats with diabetes, obesity, hypertension and older age are recently being employed in stroke studies. SenescenceAccelerated Mouse (SAM) strains suffering from early onset of age-related diseases and that demonstrate other features consistent with aging represent a quicker and more costeffective method (3). Zucker rats that are insensitive to satiety signals relayed to the hypothalamus' ventromedial nucleus, have attracted some attention because of the implications of obesity (37). Three models of diabetes, the Israeli Sand Rat, Otsuka Long-Evans Tokushima Fatty (OLETF) rat, and Ventromedial Hypothalamus Lesion (VMH) Dietary Obese Rat represent different characteristics of diabetes (3). The stroke-prone spontaneously hypertensive rat (SHRSP) is a good genetic model for particular types of stroke. These rats develop stroke even when placed on normal diets because of their genetic susceptibility to stroke (31).

However, the above-mentioned models represent individual risk factors and a more comprehensive model that incorporates all of these pathologies is needed. The term "metabolic syndrome" is used to denote constellation of various abnormalities such as obesity, elevated blood pressure, diabetes, and hyperlipidemia. These factors are supposed to be interacting components of the same single syndrome. Pathophysiology of stroke involves several complex processes running in parallel, associated with the metabolic syndrome. For instance, SHR/NDcp, a transgenic rat model spontaneously develops hypertension, obesity, hyperlipidemia and diabetes (24). Establishment of neuroprotective strategies against stroke requires more models to isolate the important pathways.

It has also been suggested that preclinical testing is inadequate for many neuroprotectants in different models, species, and time windows. It would certainly be difficult to justify the effectiveness of an agent that reduces infarct volumes in animal models with inappropriate time windows for human studies (9).

The design of clinical trials requires information about the drugs' therapeutic time windows, dose-response relationships and side effects. Moreover, futility of the drugs should also be predicted by animal studies as well as their effectiveness (12).

It is certain that some discrepancies are related to methodological shortcomings. Different methodological parameters interact to pose a dilemma of confounding results. Hypoxia tolerance and the ability of neural regeneration show differences between species. Rats, as the most widely-used animals in experimental studies, do not resemble many characteristics of stroke patients. Another complicating factor is the often-overlooked neuroprotective effects of anesthesia. There are several anesthetics that can show neuroprotective effects and reduce the number of coma days in real patients (8). We can readily assume that treatment groups receive higher dose of anesthesics because of longer procedure time. This is an important fact that prevents proper evaluation from the beginning. Establishment of accurate physiological monitoring is almost impossible, no matter how hard we try. Administration of a large amount of liquids would decrease the brain temperature and cause hypothermia that is also neuroprotective. Furthermore, the blood would become diluted, and its $\mathrm{pH}$ and the dispersion of the solutes in plasma would change (40). We suppose that most of the studies fail to meet suggested criteria of STAIR (Stroke Treatment Academic Industry Roundtable) and do not document physiological changes correctly.

Although ithas been a popular procedure, MCAO has limitations such as variability of the infarcted area's location and size (14). It has always been highly controversial if these models are really successful or not. There is no technique to evaluate if an infarct is actually formed or the MCA is completely blocked. By working on data from 502 control groups described in 346 articles, Ström et al. have investigated the method parameters' impact on mortality and variability. Their metaanalysis has revealed that the Wistar strain and intraluminal filament procedure using a silicone-coated filament resulted in smallest infarct size variability (36).

Based on the findings of Positron emission tomography (PET) scan studies, it is understood that neuroprotective agents can reduce the size of the infarction marginally without early reperfusion (23). It was revealed that the largest proportion of the final infarct (mean, 70\%) consisted of a critically hypoperfused area (17). Neuroprotectants can therefore reduce the size of the final infarct by $15 \%$, while they seem to prevent the maturation of the ischemic penumbra by half in animal models (23). However, Kaste argues that animal models will not be able to contribute to emergency stroke care without thrombolysis or other neuroprotective therapies (23).

\section{CONCLUSION}

Animal modelling definitely contributes to our knowledge of cerebral ischemia. However, we know beyond doubt that there are certain problems in the design of experimental studies. In this review, we have tried to draw attention to discrepancies between animal experiments and clinical trials. It is obvious that researchers have a great obligation to assess validity of animal models of stroke. We must always remember that we owe these creatures because the life expectancy of humans has extended by approximately 20 years due to the results obtained from animal experiments. 


\section{- REFERENCES}

1. Bailey EL, McCulloch J, Sudlow C, Wardlaw JM: Potential animal models of lacunar stroke: A systemic review. Stroke 40:e451-e458, 2009

2. Boudreau DM, Guzauskas GF, Chen E, Lalla D, Tayama D, Fagan SC, Veenstra DL: Cost-effectiveness of recombinant tissue-type plasminogen activator within 3 hours of acute ischemic stroke: Current evidence. Stroke 45(10):3032-3039, 2014

3. Brandon PL, Turner RC, Lucke-Wold AN, Rosen CL, Huber JD: Age and the metabolic syndrome as risk factors for ischemic stroke: Improving preclinical models of ischemic stroke. Yale J Biol Med 85: 523-539, 2012

4. Clancy B, Darlington RB, Finley BL: Translating developmental time across mammalian species. Neuroscience 105:7-17, 2001

5. Del Bene A, Markin SDJ, Doubal FN, Inzitari D, Wardlaw JM: Variation in risk factors for recent small subcortical infarcts with infarct size, shape and location. Stroke 44:3000-3006, 2013

6. Derrick M, Luo NL, Bregman JC, Jilling T, Ji X, Fisher K, Gladson CL, Beardsley DJ, Murdoch G, Back SA, Tan S: Preterm fetal hypoxia-ischemia causes hypertonia and motor deficits in the neonatal rabbit: A model for human cerebral palsy? J Neurosci 24: 24-34, 2004

7. Di Napoli VA, Huber JD, Houser K, Li X, Rosen CL: Early disruptions of the blood- brain barrier may contribute to exacerbated neuronal damage and prolonged functional recovery following stroke in aged rats. Neurobiol Aging 29(5):753-764, 2008

8. Donnan GA: The 2007 Feinberg lecture: A new road map for neuroprotection. Stroke 39: 242, 2008

9. Donnan GA, Davis SM: Stroke drug development usually but not always animal models. Stroke 36:2326, 2005

10. Fan X, Qiu J, Yu Z, Dai H, Singhal AB, Lo EH, Wang X: A rat model of studying tissue-type plasminogen activator thrombolysis in ischemic stroke with diabetes. Stroke 43: 567570, 2012

11. Fisher M, Feuerstein G, Howells DW, Hurn PD, Kent TA, Savitz SI, Lo EH: Update of the stroke Therapy Academic Industry Roundtable Preclinical Recommendations. Stroke 40:22442250, 2009

12. Fisher $M$, Tatlisumak $\mathrm{T}$ : Use of animal models has not contributed to development of acute stroke therapies: Con. Stroke 36(10):2324-2325, 2005

13. Franceschi C, Bonafe M, Valensin S, Olivieri F, De Luca M, Ottaviani E, De Benedictis G: Inflamm-aging. An evolutionary perspective on immunosenescence. Ann NY Acad Sci 908:244-254, 2000

14. Garcia JH, Wagner S, Liu KF, Hu XJ: Neurological deficit and extent of neuronal necrosis attributable to middle cerebral artery occlusion in rats. Statistical validation. Stroke 26: 627634, 1995

15. Garcia JH: Experimental ischemic stroke: A review. Stroke $15: 5-14,1984$
16. Gonzalez H, Hunter CJ, Bennet L, Power GG, Gun AJ: Cerebral oxygenation during postasphyxial seizures in near-term fetal sheep. J Cereb Blood Flow Metab 25:911-918, 2005

17. Harris AP, Helou S, Gleason CA, Traysman RJ, Koehler RC: Fetal cerebral and peripheral circulatory responses to hypoxia after nitric oxide synthase inhibition. Am J Physiol 281: R381-R390, 2001

18. Heiss WD, Thiel A, Ground M, Graf R: Which targets are relevant for therapy of acute ischemic stroke? Stroke 30:14861489, 1999

19. Herson PS, Koerner IP, Hurn PD: Sex, sex steroids, and brain injury. Semin Reprod Med 27: 229-239, 2009

20. Hossmann KA: Animal models of cerebral ischemia. I. Review of literature. Cerebrovasc Dis 1: 2-15, 1991

21. Howells DW, Porritt MJ, Rewell SJ, O'Collins V, Sena ES, van der Worp HB, Traystman RJ, Macleod MR: Different stroke for different folks: The rich diversity of animal models of focal cerebral ischemia. J Cereb Blood Flow Met 30:1412-1431, 2010

22. Inagaki T, Etgen AM: Neuroprotective action of acute estrogens: Animal models of brain ischemia and clinical implications. Steroids 78:597-606, 2013

23. Kaste $M$ : Use of animal models has not contributed to development of acute stroke therapies: Pro. Stroke 36(10):2323-2324, 2005

24. Kawai K, Sakairi T, Harada S, Shinozuka J, Ide M, Sato H, Tanaka M, Toriumi W, Kume E: Diet modification and its influence on metabolic and related pathological alterations in the SHR/NDmcr-cp rat, an animal model of the metabolic syndrome. Exp Toxicol Pathol 64(4):333-338, 2012

25. Ke Z, Yip SP, Li L, Zheng XX, Tong KY: The effects of voluntary, involuntary, and forced exercises on brain-derived neurotrophic factor and motor function recovery: A rat brain ischemic model. PLoS One 6: e16643, 2011

26. Koellhoffer EC, McCullough LD: The effects of estrogen in ischemic stroke. Transl Stroke Res 4:390-401,2012

27. Krakauer JW, Carmichael ST, Corbett D, Wittenberg GF: Getting neurorehabilitation right: What can be learned from animal models? Neurorehabil Neural Repair 26:923-931, 2012

28. Lekic T, Ani C: Posterior circulation stroke: Animal models and mechanisms of disease. J Biomed Biotechnol 2012:587590, 2012.

29. Lindner MD, Gribkoff VK, Donlan NA, Jones TA: Long-lasting functional disabilities in middle-aged rats with small cerebral infarcts. Neurosci 23:10913-10922, 2003

30. Macleod MR, O'Collins T, Howells DW, Donnan GA: Pooling of animal experimental data reveals influence of study design and publication bias. Stroke 35:1203-1208, 2004

31. Nabika T, Cui Z, Masuda J: The stroke-prone spontaneously hypertensive rat: How good is it as a model for cerebrovascular diseases? Cell Mol Neurobiol 24(5):639-646, 2004

32. Northington FJ: Brief update on animal models of hypoxic ischemic encephalopathy and neonatal stroke. ILAR Journal 47:32-38, 2006

33. Pedder $\mathrm{H}$, Vesterinen HM, Macleod MR, Wardlaw JM: Systematic review and meta-analysis of interventions tested in animal models of lacunar stroke. Stroke 45:563-570, 2014 
34. Schmidt A, Wellmann J, Schilling M, Strecker JK, Sommer C, Schabitz WR, Diederich K, Minnerup J: Meta-analysis of the efficacy of different training strategies in animal models of ischemic stroke. Stroke 45:239-247, 2014

35. Song Y-M, Sung J, Davey Smith G, Ebrahim S: Body mass index and ischemic and hemorrhagic stroke. A prospective study in Korean men. Stroke 35:831-836, 2004

36. Ström JO, Ingberg E, Theodorsson A, Theodorsson E: Method parameters' impact on mortality and variability in rat stroke experiments: A meta-analysis. BMC Neuroscience 14:41, 2013

37. Subramanian R, MacLeod KM: Age-dependent changes in blood pressure and arterial reactivity in obese zucker rats. Eur J Pharmacol 477(2):143-152, 2003
38. Tajiri N, Dailey T, Metcalf C, Mosley YI, Lau T, Staples M, van Loveren H, Kim, SU, Yamashima T, Yasuhara T, Date I, Kaneko Y, Borlongan CV: In vivo animal stroke models: A rationale for rodent and non-rodent human primate models. Trans Stroke Res 4(3):308-321, 2013

39. Tasdemiroglu E, Kutman MN: Pathophysiology of cerebral ischemia. Hacettepe Medical Journal 22:169-181, 1989

40. Xu SY, Pan SY: The failure of animal models of neuroprotection in acute ischemic stroke to translate to clinical efficacy. Med Sci Monit Basic Res 19: 37-45, 2013

41. Yoshida M, Yamashima T, Zhao L, Tsuchiya K, Kohda Y, Tonchev AB, Matsuda M, Kominami E: Primate neurons show different vulnerability to transient ischemia and response to cathepsin inhibition. Acta Neuropathol 104:267-272, 2002 